\title{
Simultaneous Two-color Lasing in a Single CdSSe Heterostructure Nanosheet (Invited Paper)
}

\author{
F Fan, ${ }^{\dagger} \mathrm{Z}$ Liu, ${ }^{\dagger}$ L Yin, $\mathrm{P}$ L Nichols, H Ning, S Turkdogan and C Z Ning ${ }^{*}$ \\ School of Electrical, Computer, and Energy Engineering, Arizona State University, Tempe, \\ Arizona 85287, USA \\ ${ }^{\dagger}$ These authors contributed equally to this work. \\ *ning@asu.edu, http://nanophotonics.asu.edu
}

\begin{abstract}
The ability of a single monolithic semiconductor structure to emit or lase in a broad spectrum range is of great importance for many applications such as solid state lighting and multi-spectrum detection. But spectral range of a laser or light emitting diode made of a given semiconductor is typically limited by its emission or gain bandwidth. Due to lattice mismatch, it is typically difficult to grow thin film or bulk materials with very different bandgaps in a monolithic fashion. But nanomaterials such as nanowires, nanobelts, nanosheets provide a unique opportunity. Here we report our experimental results demonstrating simultaneous lasing in two visible colors at $526 \mathrm{~nm}$ and $624 \mathrm{~nm}$ from a single CdSSe heterostructure nanosheet at room temperature. The $97 \mathrm{~nm}$ wavelength separation of the two colors is significantly larger than the gain bandwidth of a typical single II-VI semiconductor material. Such lasing and light emission in a wide spectrum range from a single monolithic structure will have important applications mentioned above.
\end{abstract}

PACs Code: Semiconductor Lasers (42.55.Px); Nanosheets (62.23.Kn); Heterostructures (79.60.Jv)

\section{Introduction}

Realization of monolithic semiconductor structures with multiple or a wide range of bandgaps have many important applications in solid state lighting, high efficient solar cells, and multi-spectral detection. In solid state lighting applications, a single semiconductor structure or device capable of emitting or lasing in the entire visible range $(400-700 \mathrm{~nm})$ would have a fundamental impact to the ultimate solution of efficient lighting. It was recently demonstrated [1] that four lasers of discrete spectral lines are virtually indistinguishable from state of the art continuous-spectrum white reference illuminant, thus demonstrating the feasibility of laser lighting. In addition, lasers can be much more efficient than light emitting diodes (LEDs). Similarly, discrete multicolor lasers have many other applications such as full-color laser display [2], white laser illumination [1], and biological inspection [3]. Even though the experimental demonstration was carried out with four separate lasers [1], a much more preferred approach would be to have all lasers of different colors produced on a same platform in a compact fashion. But achieving such emission or lasing in a wide spectrum is fundamentally challenging for several reasons. First, typical semiconductors of a given composition under reasonable excitation conditions have a gain bandwidth less than $30 \mathrm{~nm}$ [4]-[12]. Wavelength tunability of a single mode, or mode separation of multiple modes, is thus limited by such a bandwidth. Second, to have emission bandwidth significantly broader than that of a 
single-composition material, heterogeneous integration or growth of different semiconductors is necessary. Due to the large variation or significant mismatch of the lattice constants for semiconductors with distinct material compositions, growth of such structures on a single substrate becomes extremely difficult. Finally, even such materials could be grown, it is still a challenge to design an appropriate cavity structure to support wavelengths that are widely separated. This is because the short-wavelength light will be strongly absorbed by the narrow gap semiconductors involved, such that only the long-wavelength light can eventually reach the threshold and become lasing. Various attempts have been made recently to overcome these challenges and to extend the spectral range of light emission and lasing beyond the gain bandwidth of a single material. Multi-color or dual-color lasing has been observed by placing multiple nanowires within a microfiber [13], in multi-section quantum dot distributed feedback lasers [14], or by using fast-switching of different dyes in a microfluidic channel [15]. However these multicolor lasers are either achieved in separated devices, or with wavelength separation still limited within the gain bandwidth of a single semiconductor material.

In this paper, we report the first experimental observation of simultaneous lasing in two visible colors (at 526 and $624 \mathrm{~nm}$, respectively) from a single monolithic semiconductor structure. The wavelength separation of two lasing colors of $97 \mathrm{~nm}$ is much larger than the gain bandwidth of a single composition semiconductor material. The unique structure in this paper that enabled such dual wavelength lasing is a heterostructure nanosheet or nanobelt of the ternary semiconductor CdSSe produced in a single monolithic growth. Our results were enabled by some unique quality of nanowires and nanobelts that have been the focus of light emission and lasing research for the last 10 years [16]-[24]. Semiconductor nanowires and nanobelts grown using the metal-catalyzed vapor-liquid-solid mechanism have opened new possibilities for multi-color emission or lasing [17]-[22]. Even though semiconductor nanoparticles can also achieve multi-color emissions, but it is typically challenging to spatially address or control each individual color, since they are typically mixed uniformly. In addition, eventual electrical injection is needed for such applications and the poor conductivity in nanoparticles make electrical injection very inefficient. One of the important features of nanowires and nanobelts for our purpose is the ability to grow different materials with different lattice constants without introducing too many defects to degrade optical properties. This property has been utilized in the past to grow materials with very different or graded alloy compositions [18]-[21] to achieve light emission in a wide range, mostly from different wires and more recently from a single wire [25]. While we recently have demonstrated multi-color lasing from many wires on a single substrate [18], but so far, multi-color lasing from a single nanowire or belt has not been demonstrated, especially when the wavelength separation is wider than the gain bandwidth.

\section{Sample Growth and Characterization}

The CdSSe lateral heterostructure nanosheets were grown through a chemical vapor deposition (CVD) approach using CdS and CdSe powders (Alfa Aesar, 99.99\% purity) as source materials. Si substrate with $10 \mathrm{~nm}$ Au film as catalyst was used for the growth. The reactor was first evacuated to a pressure below 0.2 Torr by a mechanical pump. $150 \mathrm{sccm} \mathrm{N}_{2}$ was then introduced into the system, and flow was maintained for $30 \mathrm{~min}$ prior to growth. In the first step of the growth, only CdS powder was thermally evaporated at $880^{\circ} \mathrm{C}$ for 1 hour. Subsequently, CdSe source was introduced into the furnace, while the temperature was gradually lowered to $840^{\circ} \mathrm{C}$ and held for $40 \mathrm{~min}$ to grow the CdSe-rich sections. The growth processes is similar to that of Ref. [26], but we reversed the growth sequence to grown CdS first. Such sequential growth ensures a wide CdS-rich center section surrounded by CdSe-rich edges (see Fig.1B). The large width of CdS-rich sections is especially significant as it needs to be wide enough to achieve sufficient optical confinement for the shorter wavelength lasing modes. 


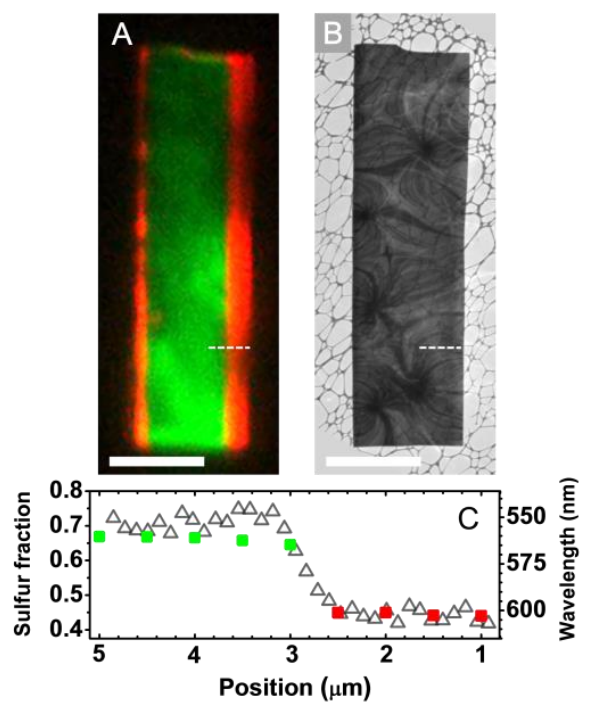

Figure 1 (A) Real-color PL image of a CdSSe lateral heterostructure nanosheet excited by 405 continuous-wave laser. (B) TEM image of the same nanosheet. The scale bars in (A) and (B) are $10 \mu \mathrm{m}$. (C) Comparison of sulfur molar fraction between EDS scan (hallow triangles) and micro-PL scan (solid squares) along the white dashed line in (A) and (B).

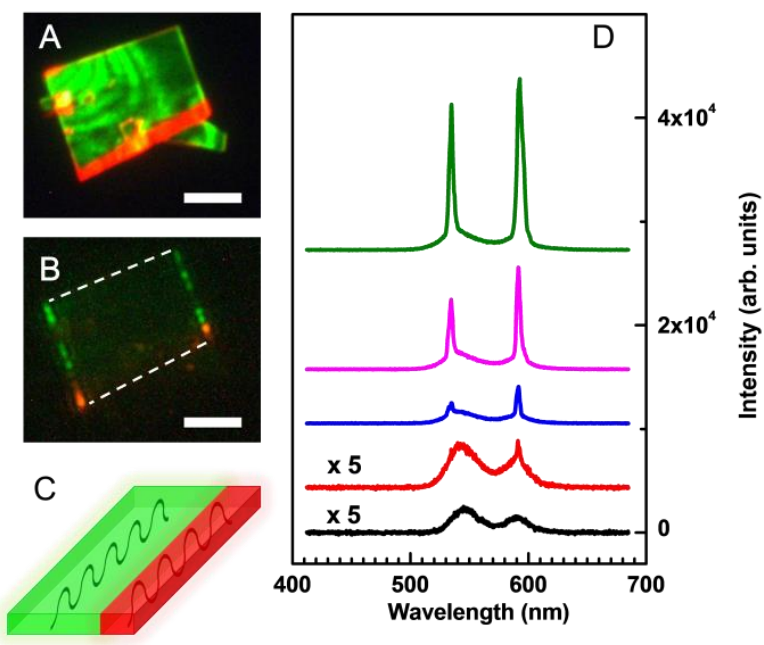

Figure 2 Real-color PL image of the nanosheet under low pumping power density at room temperature (A) and under $409 \mathrm{~kW} / \mathrm{cm}^{2}$ at $77 \mathrm{~K}(\mathrm{~B})$. The dashed lines in (B) denote the side edges of the nanosheet. The scale bars in (A) and (B) are $10 \mu \mathrm{m}$. (C) Schematic diagram of the nanosheet waveguide structures. (D) $\mathrm{PL}$ spectra at $77 \mathrm{~K}$ under increasing levels of pumping power density of 77,173 , 241,338 and $668 \mathrm{~kW} / \mathrm{cm}^{2}$ from bottom to top.

The composition distribution on the CdSSe heterostructure nanosheet is shown in Fig. 1. Fig. 1A shows the real color photoluminescence (PL) image of the heterostructure nanosheet under $405 \mathrm{~nm}$ continuous-wave (CW) laser excitation. This nanosheet has width of $11.2 \mu \mathrm{m}$ and length of $41.4 \mu \mathrm{m}$. As seen in the image, the nanosheet consists of three stripes with green emission in the center (grown initially with CdS-rich composition) and red/orange emission on both edges (grown subsequently with CdSe-rich composition). Clearly the green stripe and the wider red/orange stripe form two rectangular, coupled cavities in a side-by-side arrangement. With proper end facets, the three stripes can form three coupled cavities capable of supporting various propagating modes along the length direction. Of particular interest are the two wider stripes (the green one in the center and the red one on the right side) which form two rectangular, coupled cavities in a side-by-side arrangement, as also schematically shown in Fig. 2C. We performed micro-PL measurements by scanning the focused laser beam with a $1 \mu \mathrm{m}$ across the width of the nanosheet along the dashed line in Fig. 1A. The PL peak positions vary from green emission at $559 \mathrm{~nm}$ to orange emission at $602 \mathrm{~nm}$ with a transition occurring roughly at 3 micron in Fig.1C. Assuming the PL emission is from ternary $\mathrm{CdS}_{x} \mathrm{Se}_{1-x}$, we use the interpolation formula [27]

$$
E_{g}\left(\mathrm{CdS}_{x} \mathrm{Se}_{1-x}\right)=x E_{g}(\mathrm{CdS})+(1-x) E_{g}(\mathrm{CdSe})
$$

to fit the PL peak and to extract the alloy composition. The result is plotted in Fig.1C, where the sulfur fraction along the scanning path with transition from $45 \%$ to $67 \%$. To further confirm the composition distribution across the heterojunction, the energy dispersive X-ray spectroscopy (EDS) in a transmission electron microscope (TEM) is also performed along the same path as the PL scan. Fig. 1B shows the TEM image of this nanosheet. The image was collected with a JEOL JEM-2010 High-Resolution TEM at $200 \mathrm{kV}$, equipped with a Link energy dispersive X-ray spectroscopy (EDS) detector. The sulfur compositions obtained from EDS line scan along the dashed line are plotted in Fig. 1C, consistent with the values from micro-PL scanning.

\section{Experimental and Simulation Results}

Fig. 2A shows a PL image of another lateral heterostructure nanosheet. The dimensions of this nanosheet are $25.0 \mu \mathrm{m}$ (length) by $17.4 \mu \mathrm{m}$ (width) by $200 \mathrm{~nm}$ (thickness). The nanosheet was cleaved 
from a longer piece by the bend-to-fracture method [28]. With this procedure, high-quality end facets were created to serve as facet reflectors and to define the laser cavities. The widths of the CdS-rich (green) and CdSe-rich (orange) sections are $14.8 \mu \mathrm{m}$ and $2.6 \mu \mathrm{m}$, with PL emission peaks at $546 \mathrm{~nm}$ (green) and 591 $\mathrm{nm}$ (orange), respectively. As shown in the PL image, there are broken nanosheet debris surrounding the nanosheet of interest, observable by the lighter orange areas within the belt and the excess green areas outside of the nanosheet perimeter. To demonstrate the capability of dual-color lasing, the sample was transferred onto a sapphire substrate and loaded into a cryostat for low temperature measurement. We optically pumped the nanosheet by the third harmonic of a Q-switched Nd:YAG laser $(355 \mathrm{~nm}, 10 \mathrm{~Hz}, 9$ $\mathrm{ns})$. The laser was focused into a $120 \mu \mathrm{m}$ by $60 \mu \mathrm{m}$ elliptical beam at an angle of $60^{\circ}$ from the sample normal direction. The real-color image of single pulse induced lasing at higher pumping level at $77 \mathrm{~K}$ is shown in Fig. 2B. As can be seen clearly, the largely uniform spontaneous emission of Fig. 2A is now replaced by the green and orange bright spots along the end facets, signifying the occurrence of lasing. Due to the gain-guiding effect and the refractive index contrast, green and orange emission can be well-separated and confined in their own cavities, which is schematically shown in Fig. 2C. This is reflected in Fig. 2B, where green and red emissions from the end facets are from the respective segments of CdS-rich or CdSe-rich materials. The interaction between the green and orange modes is minimized in this structure. The side-by-side arrangement is an important geometry for reaching the respective lasing thresholds of both colors. This is in contrast to structures where bandgap changes along the length of a nanowire [29], where short-wavelength emission from the wide bandgap region (CdS-rich) can be significantly absorbed by the narrow bandgap region (CdSe-rich). Such absorption will increase the threshold for the short wavelength lasing, very often to a degree that is too high to achieve lasing. Our side-by-side arrangement minimizes such undesired absorption and allows the respective lasing thresholds of both wavelengths to be reached.

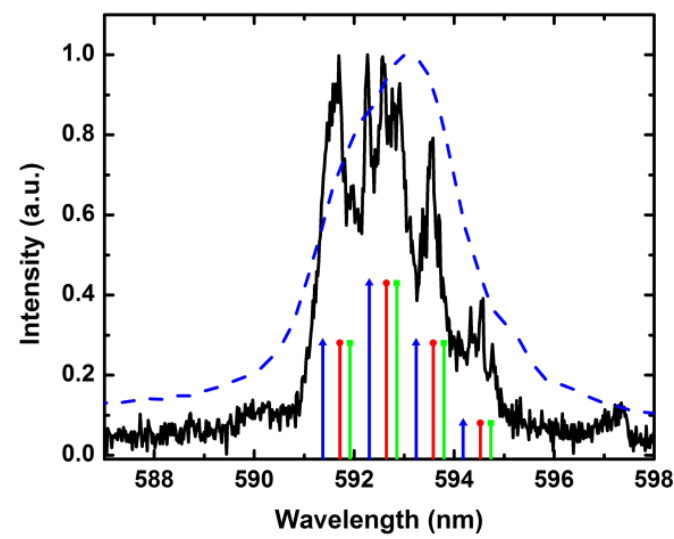

Figure 3 High resolution (black solid) and low resolution (blue dash) spectra for the orange lasing peak pumped at $424 \mathrm{~kW} / \mathrm{cm}^{2}$. The green, red and blue vertical lines represent the wavelengths of $\mathrm{TE}_{10}$, $\mathrm{TE}_{20}$ and $\mathrm{TE}_{30}$ transverse modes. Each transverse mode has 4 associated longitudinal modes. The intensity of vertical lines of each group is calculated by the Gaussian convolution.

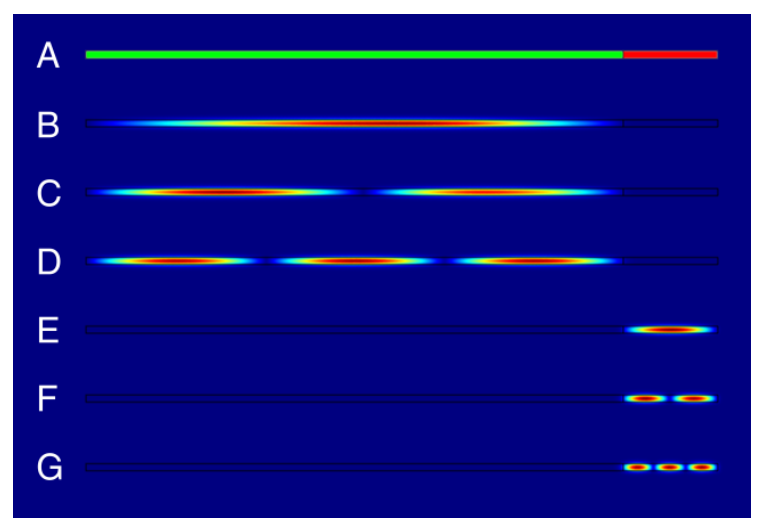

Figure 4 2D Simulation of the optical modes of the CdSSe heterostructure nanosheet. The figure shows the cross section of a nanosheet in the thickness-width plane. The propagation direction is perpendicular to the paper plane. (A) The schematic of the nanosheet cross-section. The green (left) and red (right) rectangles represent the CdS-rich (14.8 $\mu \mathrm{m}$ width) and CdSe rich segments (2.6 $\mu \mathrm{m}$ width), respectively. The thickness is $200 \mathrm{~nm}$. (B-D): The first three modes around $534 \mathrm{~nm}$. (E-G): The first three modes around $592 \mathrm{~nm}$.

Fig. 2D shows the PL spectra under excitation of a single pulse with increasing pump power density. At lower pumping levels, only broadband spontaneous emission from the CdS-rich and CdSe-rich sections is observed. With increasing pump pulse power, a narrow peak appears at $592 \mathrm{~nm}$ at an excitation level of $173 \mathrm{~kW} / \mathrm{cm}^{2}$, indicating the onset of lasing for the orange color in the CdSe-rich section. The same is observed for green color lasing at $534 \mathrm{~nm}$ in the CdS-rich section when the excitation level reaches 241 $\mathrm{kW} / \mathrm{cm}^{2}$. The $58 \mathrm{~nm}$ separation of the two lasing peaks is much larger than the gain bandwidth of a typical $\mathrm{Zn}-\mathrm{Cd}-\mathrm{S}-\mathrm{Se}$ semiconductor material system including $\mathrm{CdS}$ or CdSe [2]-[8]. The narrowest linewidths of 
the two colors in Fig. 2D are around $4 \mathrm{~nm}$, which are significantly broader than the typical linewidth of a single mode semiconductor laser. Detailed examination proves such broad linewidths of both sections under higher excitation intensities is due to the well-known multimode lasing behavior [30]-[35] and spectrometer resolution limitation (at $0.1 \mathrm{~nm}$ ). To confirm this, we performed high resolution measurement for the orange lasing peak and compared with the low resolution measurement (shown in Fig. 3) to examine the detailed mode features. The high resolution measurement reveals that the wide peak in low resolution measurement actually consists of multiple peaks with individual linewidths as narrow as $0.1-0.3$ $\mathrm{nm}$. The multiple lasing peaks can be divided into four groups and each group consists of a series of closely spaced peaks. The four groups of lasing peaks can be identified as the longitudinal modes of different orders with each group consist of several closely spaced transverse modes. The wavelengths of three transverse modes $\mathrm{TE}_{10}, \mathrm{TE}_{20}, \mathrm{TE}_{30}$ and the corresponding longitudinal modes are also calculated and labeled in Fig. 3 for comparison. The mode spacing between the transverse modes is typically smaller than the longitudinal mode separation and can be as small as $0.2 \mathrm{~nm}$, which almost reaches the resolution limitation of our spectrometer system. We attribute the multimode lasing features to the large size of the nanosheet structures in both directions, as compared to the wavelengths. With increasing pump intensity, more modes are excited above the threshold (see Appendix for another nanowire case). Their close proximity to each other results in broader peaks as observed in low resolution measurements.

In order to demonstrate spatial separation of the green and orange lasing modes in the side-by-side cavities of the heterostructure nanosheet, we performed a 2D simulation for the optical modes at the lasing wavelengths of $534 \mathrm{~nm}$ and $592 \mathrm{~nm}$, as observed experimentally (see Fig. 2D). In this 2D model, the length of the nanosheet is assumed to be infinite long with cross-section as shown in Fig. 2C. The sulfur compositions $(x)$ of the CdS-rich and CdSe-rich sections as determined from their PL emission peaks are $x$ $=0.82$ and $x=0.49$, respectively. The complex refractive indices of these CdSSe alloys are listed in Table 1. The real parts of the refractive indices for $\mathrm{CdS}_{0.82} \mathrm{Se}_{0.18}$ and $\mathrm{CdS}_{0.49} \mathrm{Se}_{0.51}$ are obtained from Ref. [36] and refractive index database SOPRA [37], respectively. The gain and absorption are taken into account by the imaginary part of the complex refractive index. The gain $(g)$ or absorption obeys the following relationship:

$$
g=\frac{4 \pi}{\lambda} k
$$

where $k$ is the imaginary part of the complex index of refraction, $\lambda$ is the wavelength, and $g$ is the gain or absorption coefficient (with a negative sign). A positive extinction coefficient represents gain, and negative represents absorption. The absorption of $\mathrm{CdS}_{0.49} \mathrm{Se}_{0.51}$ at $534 \mathrm{~nm}$ is $50,000 \mathrm{~cm}^{-1}$ based on the calculations and data in Ref. [38]. Under optical pumping, the gain coefficients in active regions are assumed to be $3,000 \mathrm{~cm}^{-1}$ which is reasonable assumption to overcome the mirror loss.

Table 1 Summary of Refractive Index used in Simulation

\begin{tabular}{ccc}
\hline & \multicolumn{2}{c}{ Refractive Index } \\
\cline { 2 - 3 } Wavelength & $\mathrm{CdS}_{0.82} \mathrm{Se}_{0.18}$ & $\mathrm{CdS}_{0.49} \mathrm{Se}_{0.51}$ \\
$534 \mathrm{~nm}$ & $2.625+0.0127 \mathrm{i}$ & $2.738-0.213 \mathrm{i}$ \\
$592 \mathrm{~nm}$ & 2.538 & $2.837+0.0141 \mathrm{i}$ \\
\hline
\end{tabular}

From the simulation, the first three modes with highest gain are selected for each wavelength. The amplitude of the electric fields of these modes at $534 \mathrm{~nm}$ and $592 \mathrm{~nm}$ are shown in Fig.4B-D and Fig. $4 \mathrm{E}-\mathrm{G}$, respectively. For both wavelengths, the highest-gain modes are well-confined in their respective active regions (CdS-rich segment for $534 \mathrm{~nm}$, left in Fig. 4A, and CdSe-rich segment for $592 \mathrm{~nm}$, right in Fig. 4A). It is important to determine the confinement factor, defined as the ration of the modal gain to the material gain [39],[40], as it is known now that it can be significantly large in nanowires and nanobelts [30],[39],[40]. The confinement factors for all of these modes are slightly larger than unit at 1.03. In general, modes of both wavelengths can be confined in either the CdS-rich or CdSe-rich segment due to the contrast between the refractive indices. However, only the modes in appropriate gain medium with wavelength insider the gain bandwidth have the possibility to lase. As a result, high gain modes at $534 \mathrm{~nm}$ 
(or $592 \mathrm{~nm}$ ) can only lase in the CdS-rich (or CdSe- rich) active regions. The refractive index contrast and the gain confinement ensure that the modes of both wavelengths are confined within their respective gain media without much leakage to the lossy regions. This is especially important for the green modes, so that they will not be subject to too strong absorption by the narrow gap material of the CdSe-rich side. The simulation proves our previous statement that the CdSSe heterostructure nanosheet provides an appropriate structure to support dual-wavelength lasing in a single structure.

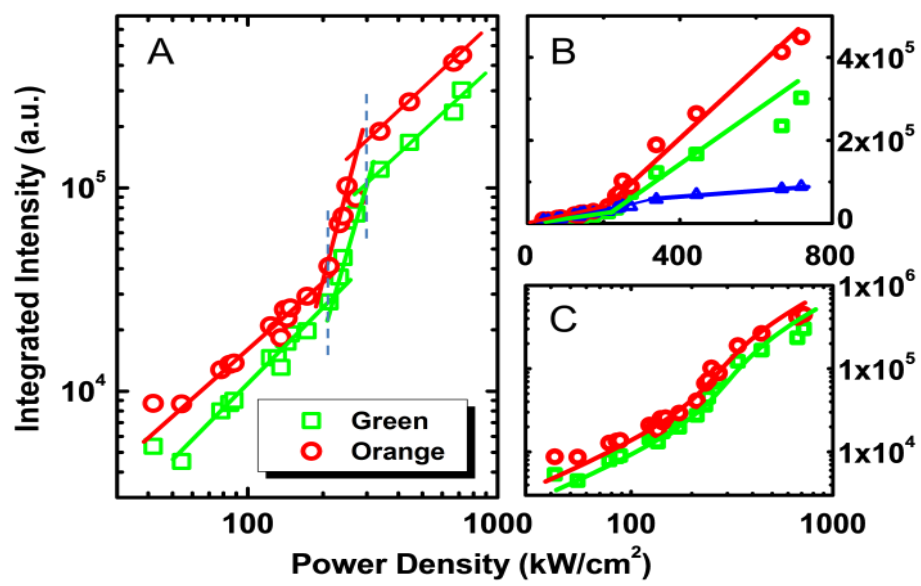

Figure 5 (A) Output intensities of the two lasing colors versus pumping power density at $77 \mathrm{~K}$ in double-log scale. The three straight lines indicate the slopes of three regions. (B) Same as in (A), but on linear scale, where we also show the spontaneous emission (blue color with triangles) with a strong saturation after the threshold. (C) Same as in (A), but shows the fitting with the multi-mode laser model, as explained in the text.

The integrated light intensity is plotted as function of pumping power density on both $\log -\log$ (Fig. 5A) and linear scales (Fig. 5B) to corroborate the spectral features and to further establish the lasing behavior at two distinct wavelengths. Shown in the Fig. 5B, the lasing intensity of both colors increase linearly with pump power after threshold $\left(260 \mathrm{~kW} / \mathrm{cm}^{2}\right.$ for the green modes and $230 \mathrm{~kW} / \mathrm{cm}^{2}$ for the orange modes). The total spontaneous emission output increases much more slowly than the lasing modes in the high excitation region, demonstrating the typical behavior associated with density clamping (or slowly increasing) near the lasing threshold. The log-log scale of the lasing output as a function of the excitation power features the well-known S-like behavior with three significantly different regions with different slopes, as indicated by various straight lines used to extract the slopes. At low pumping, the spontaneous emission is dominant, so the mode intensities increase linearly with a slope of 1.1 for orange and 1.3 for green. Around the thresholds, the output intensities of both lasing modes show superlinear increases with slopes of 5.1 for orange and 4.7 for green. With further increase of the excitation power, the slopes of both curves return to 1.1, indicating the above-threshold lasing operation of both colors. The dependence of total intensity on pumping displays a typical threshold behavior of multimode lasing, which is somewhat more complicated than that of a single mode laser. To examine the lasing threshold behavior including the multiple transverse and longitudinal modes, experimental data of total light intensity as a function of total pump intensity (shown in Fig. 5C) is fitted by the multimode lasing model proposed by Casperson [35]. In this model, the total output power of multimode lasing is given by

$$
I_{t}=\frac{x_{0} p}{(1+x)\left[1-p(1+x)^{-1}\right]^{1 / 2}}
$$

where $p$ is the pump laser power normalized to the lasing threshold and the parameter $x$ is related to $x_{0}$ through

$$
\frac{x}{x_{0}}=\sqrt{\frac{1+x}{1+x-p}}
$$


Here $x_{0}$ is a parameter proportional to the total spontaneous emission coupled into multiple lasing modes. Mathematically $x_{0}$ is proportional to the sum of the spontaneous emission factor $\beta$ of each lasing mode. For a conventional semiconductor laser, $x_{0}$ is less than 0.001 [35]. For a semiconductor nanowire laser, $x_{0}$ becomes larger, around 0.02 [30],[33]. Fig. 5C shows the fitting result. The best fitting was obtained when $x_{0}$ was taken as 0.085 for green mode and 0.090 for orange mode. The relatively large $x_{0}$ as compared to nanowire lasers can be understood by the relationship between $x_{0}$ and spontaneous emission factor. $x_{0}=0.02$ was obtained when a clear set of longitudinal lasing modes was observed from the nanowire. In our case, the multimode lasing from the nanosheet contains transverse in addition to longitudinal modes. These transverse modes survive during the lasing process due to the large confinement factor, as previously addressed. All of these modes contribute to the spontaneous emission factor $x_{0}$ to increase its magnitude. As a result, the light-in-light-out curve of nanosheet laser shows a slightly soft threshold.
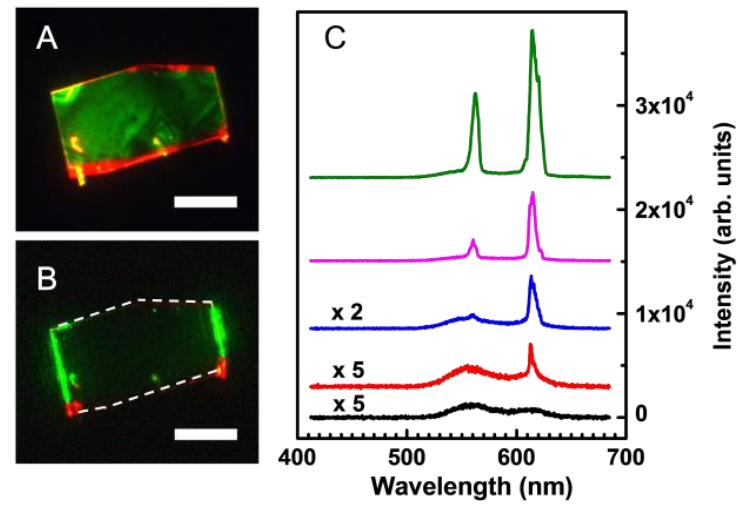

Figure 6 Real-color PL image of the nanosheet at RT under low pumping power density (A) and under 667 $\mathrm{kW} / \mathrm{cm}^{2}(\mathrm{~B})$. The scale bars in (A) and (B) are $25 \mu \mathrm{m}$. (C) PL spectra at RT under increasing levels of pumping power density of $114,184,257,310$ and $546 \mathrm{~kW} / \mathrm{cm}^{2}$ from bottom to top respectively.

Room temperature (RT) lasing has also been achieved on a larger nanosheet (66.5 $\mu \mathrm{m}$ in length, 33.0 $\mu \mathrm{m}$ in width, and $200 \mathrm{~nm}$ in thickness). As shown in Fig. 6A, the widths of the green and the wider orange stripes are around $30 \mu \mathrm{m}$ and $3 \mu \mathrm{m}$, respectively. The real color lasing image and PL spectra evolution are shown in Fig. 6B and 6C, respectively. The lasing wavelengths of the orange and green peaks are $613 \mathrm{~nm}$ and $562 \mathrm{~nm}$, respectively, with a wavelength separation of $51 \mathrm{~nm}$. The estimated threshold is $300 \mathrm{~kW} / \mathrm{cm}^{2}$ for the green modes and $227 \mathrm{~kW} / \mathrm{cm}^{2}$ for the orange modes. The lasing sequence for the two colors at RT is consistent with the result at $77 \mathrm{~K}$, both exhibiting higher thresholds for the green modes than for the orange modes. As in Fig.2, the main peaks shown in Fig.6B broadens as pumping power is increased due to the excitation of increased number of modes, as we discussed in connection with Fig. 3. 


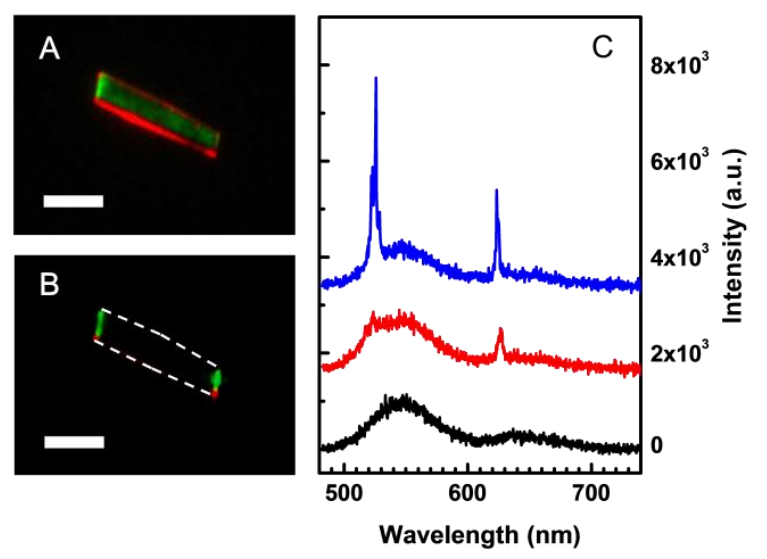

Figure 7 Real-color PL image of the nanosheet at RT by changing growth method under low pumping power density (A) and under high pumping power density (B). The scale bars in (A) and (B) are $10 \mu \mathrm{m}$. (C) PL spectra at RT under increasing levels of pumping power density of 196, 243 and $306 \mathrm{~kW} / \mathrm{cm}^{2}$ from bottom to top respectively.

The wavelength separation of two lasing colors can be further expanded by improving the growth methods of CdSSe nanosheet. The improved growing steps are the same as described in section 2 except that we totally terminated the CdS source supply before introducing of CdSe source. By doing this, the ions exchange between sulfur and selenium can be minimized during the second step of the growth and as a result, the wavelength separation of two color lasing can be further expanded. Fig. 7 demonstrates the two color lasing results of the nanosheet by changing the growth method. The lasing wavelengths are 526 $\mathrm{nm}$ and $624 \mathrm{~nm}$ respectively with a separation of $97 \mathrm{~nm}$. The long wavelength has now been fully expanded into the red color range. The dimensions of the nanosheet in Fig. 7A are $25.0 \mu \mathrm{m}$ (length) by $17.4 \mu \mathrm{m}$ (width) by $200 \mathrm{~nm}$ (thickness), much smaller than that of the nanosheets demonstrated above. As a result, less significant multimode behavior is shown in Fig. 7C. Other features are similar to previous ones.

\section{Conclusion}

To summarize, we demonstrated simultaneous lasing in two distinct visible colors from a single CdSSe lateral heterostructure nanosheet. This demonstration was made possible by utilizing an important progress made recently in the growth of nano-materials using the metal-catalyzed vapor-liquid-solid CVD growth. The key material enabler is the virtually substrate-insensitive growth of a heterostructures with wide composition variation within a single nanosheet. The side-by-side geometry overcomes one of the most challenging issues with multi-color lasing: the absorption of short-wavelength light by the narrow gap semiconductors. The dual-color lasing was established by a combination of different measurements including high and low resolution spectral measurements, integrated light intensity measurements at various pumping levels, and changes of near-field spatial profiles with pumping levels. The multimode lasing behavior has been clearly shown in high resolution measurements. In low resolution measurements, the observed linewidth broadening with increasing pump power is attributed to the increasing number of modes contributing to the overall peak width. This has been known for multimode lasing in semiconductors [30]-[35],[40]. One of the key quantities is the wavelength separation between the two colors, which is eventually expanded to $97 \mathrm{~nm}$ at RT, much larger than the typical gain bandwidth of semiconductor materials $(<30 \mathrm{~nm})$ in this wavelength range. Lasing with such widely separated colors from a single semiconductor structure can be important for many applications. 
Acknowledgement: This research is supported by Army Research Office (Grant number W911NF-08-1-0471, under Michael Gerhold).

\section{Appendix A: Experimental illustration of modes evolution with pumping}

To understand the increasing linewidth with pumping as measured with low resolution spectroscopy, we studied a CdS nanowire with multiple transverse and longitudinal modes using a high spectral resolution measurement, with the results presented in Fig. A1. Since our nanowire has a much shorter length (at 10 microns) than the lengths of those nanosheet structures studied earlier, the longitudinal modes are separated with larger spectral intervals. Therefore, it is much easier to distinguish each longitudinal mode. Similar to the lasing spectrum of the nanosheet shown in Fig. 3, the separated groups of modes represent different longitudinal modes and the fine peaks in each group are different transverse modes. When raising the pumping power, more longitudinal modes appear at longer wavelength side, from 1 in the bottom panel to 5 in the top panel. This can be explained by the gain spectrum broadening and heating effect. The linewidth of each longitudinal mode increases with pumping owing to the increasing numbers of transverse modes. This multimode lasing and linewidth broadening effect was observed in both nanowire and nanosheet [30],[41],[42].

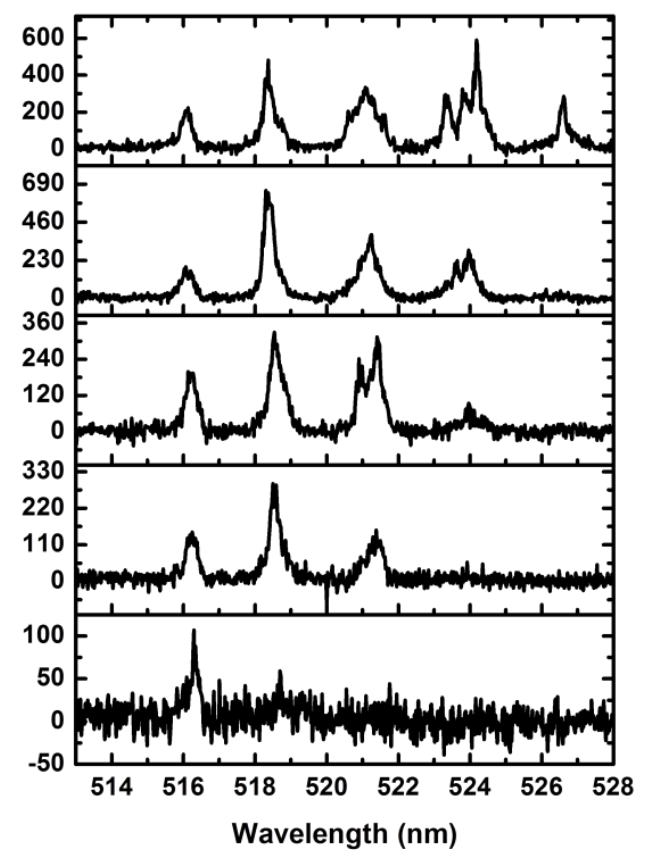

Figure A1. Lasing spectra of a CdS nanowire of $10 \mu \mathrm{m}$ in length and $0.5 \mu \mathrm{m}$ in diameter. From bottom to top panels, the pumping power densities are $0.18,0.21,0.30,0.39,0.43$ $\mathrm{MW} / \mathrm{cm}^{2}$ respectively. The numbers of longitudinal modes (represented by the broad peaks) are 1, 3, 3, 4, and 5, respectively from the bottom to top. Within each broad peak, there are many fine features representing the transverse modes. 


\section{References}

[1] Neumann A, Wierer J J, Davis W, Ohno Y, Brueck S R J, Tsao J Y 2011 Four-color laser white illuminant demonstrating high color-rendering quality Opt. Express 19 A982-90

[2] Hollemann G, Braun B, Dorsch F, Hennig P, Heist P, Krause U, Kutschki U, Hermann A V 2000 RGB lasers for laser projection displays Proc. SPIE 3954 140-51

[3] Kotani A, Witek M A, Osiri J K, Wang H, Sinville R, Pincas H, Barany F, Soper S A 2012 EndoV/DNA ligase mutation scanning assay using microchip capillary electrophoresis and dual-color laser-induced fluorescence detection Anal. Methods 4 58-64

[4] Girndt A, Koch S W, Chow W W 1998 Microscopic theory of laser gain in semiconductor quantum wells. Appl. Phys. A 66 1-12

[5] Yoshida H, Gonno Y, Nakano K, Taniguchi S, Hino T, Ishibashi A, Ikeda M, Chuang S L 1996 Gain characteristics of gainguided II-VI laser diodes Appl. Phys. Lett. $693893-5$

[6] Lougue F P, Rees P, Heffernan J F, Jordan C, Donegan J F, Hegarty J, Hiei F, Taniguchi S, Hino T, Nakano K, Ishibashi A 1998 Optical gain in (Zn, Cd)Se-Zn(S, Se) quantum wells J. Opt. Soc. Am. B 15 1295-304

[7] Girndt A, Jahnke F, Knorr A, Koch S W, Chow W W 1997 Multi-Band bloch equations and gain spectra of highly excited II-VI semiconductor quantum wells Phys. Stat. Sol. B 202 725-39

[8] Yamada Y, Masumotoa Y, Taguchi T 2002 Formation of optical gain due to exciton localization in $\mathrm{Cd}_{\mathrm{x}} \mathrm{Zn}_{1-\mathrm{x}} \mathrm{S}-\mathrm{ZnS}$ strained-layer quantum wells Physica B: Cond. Mat. 191 83-9

[9] Klingshirn C, Kalta H, Umlauffa M, Petria W, Majumdera F A, Bogdanova S V, Langbeina W, Grüna M, Hettericha M, Geyzersb K P, Heukenb M, Naumovc A, Stanzlc H, Gebhardtc W 1994 Stimulated emission of IIVI epitaxial layers J. Crys. Growth 138 786-90

[10] Motisuke P, Argüello CA, Luzzi R 1977 Effect of excited electron states lifetime on gain spectra of EHL in CdS Solid State Commun. 23 617-20

[11] Kuball M, Jeon E S, Song Y K, Nurmikko A V, Kozodoy P, Abare A, Keller S, Coldren L A, Mishra U K, DenBaars S P, Steigerwald D A 1997 Gain spectroscopy on InGaN/GaN quantum well diodes Appl. Phys. Lett. 70 2580-2

[12] Hakki B W, Paoli T L 1975 Gain spectra in GaAs double-heterostructure injection lasers J. Appl. Phys. 46 1299-306

[13] Ding Y, Yang Q, Guo X, Wang S, Gu F, Fu J, Wan Q, Cheng J, Tong L 2009 Nanowires/microfiber hybrid structure multicolor laser Opt. Express 17 21813-8

[14] Naderi N A, Grillot F, Yang K, Wright J B, Gin A, Lester L F 2010 Two-color multi-section quantum dot distributed feedback laser Opt. Express 18 27028-35

[15] Tang S K Y, Li Z, Abate A R, Agresti J J, Weitz D A, Psaltis D, Whitesides G M 2009 A multi-color fast-switching microfluidic droplet dye laser Lab Chip 9 2767-71

[16] Ning C Z 2012 Semiconductor nanowire lasers In: Coleman J J, Bryce A C, Jagadish C. Semiconductors and Semimetals (Burlington, Academic Press)

[17] Huang M H, Mao S, Feick H, Yan H, Wu Y, Kind H, Weber E, Russo R, Yang P D 2001 Room-Temperature Ultraviolet Nanowire Nanolasers Science 292 1897-99

[18] Pan A L, Zhou W, Leong E S P, Liu R, Chin A H, Zou B, Ning C Z 2009 Continuous Alloy-Composition Spatial Grading and Superbroad Wavelength-tunable nanowire lasers on a single chip Nano Lett. 9 784-8

[19] Pan A L, Liu R B, Sun M H, Ning C Z 2010 Spatial composition grading of quaternary ZnCdSSe alloy 
nanowires with tunable light emission between 350 and $710 \mathrm{~nm}$ on a Single Substrate ACS. Nano 4, 671-80

[20] Liu Y, Zapien J A, Shan Y Y, Geng C Y, Lee C S, Lee S T 2005 Wavelength Controlled Lasing in $\mathrm{Zn}_{\mathrm{x}} \mathrm{Cd}_{1-\mathrm{x}} \mathrm{S}$ Single crystal nanoribbons Adv. Mater. 17 1372-77

[21] Zapien J, Liu Y, Shan Y, Tang H, Lee C, Lee S T 2007 Continuous near-infrared-to-ultraviolet lasing from II-VI nanoribbons Appl. Phys. Lett. 90 213114-6

[22] Chin A H, Vaddiraju S, Maslov A V, Ning C N, Sunkara M K, Meyyappan M 2006 Near-infrared semiconductor subwavelength-wire lasers Appl. Phys. Lett. 88163115

[23] Duan X F, Huang Y, Agarwal R, Lieber C M 2003 Single-nanowire electrically driven lasers Nature 421 241-245

[24] Zimmler M A, Bao J, Capasso F, Muller S, Ronning C 2008 Laser action in nanowires: Observation of the transition from amplified spontaneous emission to laser oscillation Appl. Phys. Lett. 93051101

[25] Yang Z Y, Xu J Y, Wang P, Zhuang X J, Pan A L, Tong L M 2011 On-Nanowire spatial band gap design for white light emission Nano Lett. 11 5085-89

[26] Kim Y L, Jung J H, Yoon H S, Song M S, Bae S H, Kim Y, Chen Z G, Zou J, Joyce H J, Gao Q, Tan H H, Jagadish C $2010 \mathrm{CdS} / \mathrm{CdSe}$ lateral heterostructure nanobelts by a two-step physical vapor transport method Nanotechnology 21145602

[27] Kwon S J, Choi Y J, Park J H, Hwang I S, Park J G 2005 Structural and optical properties of $\mathrm{CdS}_{\mathrm{x}} \mathrm{Se}_{1-\mathrm{x}}$ nanowires Phys. Rev. B 72, 205312

[28] Tong L M, Lou J Y, Gattass R R, He S L, Chen X W, Liu L, Mazur E 2005 Assembly of silica nanowires on silica aerogels for microphotonic devices Nano Lett. 5 259-62

[29] Gu F X, Yang Z Y, Yu H K, Xu J Y, Wang P, Tong L M, Pan A L 2011 Spatial bandgap engineering along single alloy nanowires J. Am. Chem. Soc. 133 2037-9

[30] Zimmler M A, Capasso F, Muller S, Ronning C 2010 Optically pumped nanowire lasers: Invited review Semicond. Sci. Technol. 25024001

[31] O'Carroll D, Lieberwirth I, Redmond G 2007 Microcavity effects and optically pumped lasing in single conjugated polymer nanowires Nature Nanotechnology 2 182-4

[32] Johnson J C, Yan H, Yang P D, Saykally R J 2003 Optical Cavity Effects in ZnO Nanowire Lasers and Waveguides J. Phys. Chem. B 107 8816-28

[33] Oulton R F, Sorger V J, Zentgraf T, Ma R M, Gladden C, Dai L, Bartal G, Zhang X 2009 Plasmon lasers at deep subwavelength scale Nature 461 629-32

[34] Ma R M, Oulton R F, Sorger V J, Bartal G, Zhang X 2011 Room-temperature sub-diffraction-limited plasmon laser by total internal reflection Nature Material 10 110-3

[35] Casperson L W 1975 Threshold characteristics of multimode laser oscillators J. App. Phys. 46 5194-201

[36] Jensen B, Torabi A 1986 Refractive index of hexagonal II-VI compounds CdSe, CdS, and $\mathrm{CdSe}_{\mathrm{x}} \mathrm{S}_{1-\mathrm{x}} J$. Opt. Soc. Am. B 3 857-63

[37] Accessed October 18, 2012, at http://www.sspectra.com/sopra.html

[38] Gutowski J, Sebald K, Voss T 2009 Group III Condensed Matter: Volume 44B (Heidelberg, Springer Press)

[39] Maslov A V, Ning C Z 2004 Modal gain in a semiconductor nanowire laser with anisotropic bandstructrure IEEE J. Quantum Electron 40 1389-97

[40] Ning C Z 2010 Semiconductor nanolasers Phys. Status Solidi B 247 774-88

[41] Agarwal R, Barrelet C J, Lieber C M 2005 Lasing in single cadmium sulfide nanowire optical cavities Nano Lett. $5917-20$ 
[42] Cao B, Jiang Y, Wang C, Wang W, Wang L, Niu M, Zhang W, Li Y, Lee S T 2007 Synthesis and Lasing Properties of Highly Ordered CdS Nanowire Arrays Adv. Funct. Mater. 17 1501-6 